BMJ Open

Diabetes

Research

\& Care

\title{
Individual and combined contributions of age-specific and sex-specific pulse pressure and brachial-ankle pulse wave velocity to the risk of new-onset diabetes mellitus
}

\author{
Yuntao Wu, ${ }^{1}$ Xu Han, ${ }^{2}$ Jingli Gao, ${ }^{1}$ Yanxiu Wang, ${ }^{1}$ Chenrui Zhu, ${ }^{1}$ Zhe Huang, \\ Aijun Xing, ${ }^{1}$ Shuohua Chen, ${ }^{1}$ Yihan Ma, ${ }^{1}$ Mengyi Zheng, ${ }^{2}$ Qian Liu, ${ }^{1}$ \\ Yaohua Tian (D) , ${ }^{3}$ Shouling Wu (D) ${ }^{1}$
}

To cite: Wu Y, Han X, Gao J, et al. Individual and combined contributions of age-specific and sex-specific pulse pressure and brachial-ankle pulse wave velocity to the risk of new-onset diabetes mellitus. BMJ Open Diab Res Care 2021;9:e001942. doi:10.1136/ bmjdrc-2020-001942

- Supplemental material is published online only. To view, please visit the journal online (http://dx.doi.org/10.1136/ bmjdrc-2020-001942).

YWu and XH contributed equally.

Received 9 0ctober 2020 Revised 19 April 2021 Accepted 25 April 2021

Check for updates

(C) Author(s) (or their employer(s)) 2021. Re-use permitted under CC BY-NC. No commercial re-use. See rights and permissions. Published by BMJ.

For numbered affiliations see end of article.

Correspondence to Dr Shouling Wu; drwus|@163.com and Dr Yaohua Tian; yaohua_tian@hust.edu.cn

\section{ABSTRACT}

Introduction We aimed to investigate the individual and combined effects of age-specific and sex-specific pulse pressure (PP) and brachial-ankle pulse wave velocity (baPWV) on the incidence of new-onset diabetes mellitus. Research design and methods Participants in the Kailuan study cohort who were $\geq 20$ years old, participated in follow-up assessments and underwent baPWV measurements in 2010-2011, 2012-2013, and 20142015 were studied. The participants were allocated to four groups according to their PP and baPWV status, each categorized as high or normal, according to age-specific and sex-specific median values. Cox proportional hazards models were used to explore the individual and combined effects of PP and baPWV on the incidence of diabetes mellitus.

Results There were 18619 participants who were followed for $4.27 \pm 1.91$ years. A total of 877 new cases of diabetes were identified, and the incidence density was $11.03 / 1000$ per year. Using the normal PP and normal baPWV group as the reference group, the multivariableadjusted $\mathrm{HRs}$ and $95 \%$ Cls for diabetes mellitus in the high PP and high baPWV groups were 1.08 (0.93 to 1.25) and 1.64 (1.41 to 1.90), respectively. Compared with the normal PP/baPWV group, the HR and $95 \% \mathrm{Cl}$ for diabetes in the normal PP/high baPWV, the high PP/normal baPWV, and high PP/baPWV groups were 1.66 (1.35 to 2.05), 1.09 (0.86 to 1.37), and 1.74 (1.43 to 2.13 ), respectively. Conclusions High baPWV was independently associated with a higher risk of diabetes mellitus, and individuals with both high baPWV and high PP were at a still higher risk of diabetes mellitus.

\section{INTRODUCTION}

Diabetes mellitus is a chronic noncommunicable disease that is highly prevalent worldwide. A recent study estimated that there are 463 million people around the world who have diabetes and predicted that this number will reach 700 million by $2045{ }^{1}$ Because of continuing social and economic

\section{Significance of this study}

What is already known about this subject?

- Pulse pressure (PP) is an independent predictor of new-onset diabetes in hypertensive individuals.

- Brachial-ankle pulse wave velocity (baPWV) is positively associated with the risk of new-onset diabetes.

What are the new findings?

- High baPWV was positively associated with the risk of new-onset diabetes mellitus.

- High PP was not independently associated with the risk of developing diabetes.

- Participants with both high baPWV and high PP were at a still higher risk of diabetes mellitus.

How might these results change the focus of research or clinical practice?

- BaPWV is a better predictor of diabetes than PP. Therefore, the measurement of baPWV in the general population may help prevent the development of diabetes.

advances, the prevalence of this disease in China is steadily increasing. A national survey conducted in 2013 generated estimated prevalences of diabetes and pre-diabetes in China of $10.9 \%$ and $35.7 \%$, respectively. ${ }^{2}$ Diabetes is a risk factor for cardiocerebrovascular events and is associated with other complications, such as nephropathy, retinopathy, and peripheral vascular disease, which create significant social and economic burdens.

The etiology of diabetes is not yet clear. Heredity, physical inactivity, and a poor diet are well-known risk factors for diabetes. ${ }^{3}$ Arterial stiffness has also been shown to be a risk factor for diabetes in a large number of studies. Yasuno et al found that high pulse pressure (PP) is a predictor of new-onset 
diabetes in hypertensive patients, and Zhang et at $\tilde{l}^{5}$ showed that PP and brachial-ankle pulse wave velocity (baPWV) are associated with a higher risk of diabetes in hypertensive adults. Both PP and pulse wave velocity (PWV) are indicators of arterial stiffness, but the information they provide differs. ${ }^{67}$ BaPWV reflects both central and peripheral arterial stiffness, and PP only reflects central arterial stiffness, but changes in peripheral arteries do not significantly affect PP. In addition, some previous studies have shown that the individual effects of PP and PWV on the risks of cardiovascular events differ and that the two variables also have a combined effect on the incidences of such events. ${ }^{68}$

In previous studies, the associations of PP and PWV with diabetes have been separately evaluated. However, to date, the combined effect of PP and baPWV on the risk of diabetes has not been evaluated. Arterial stiffness reflects the degeneration of the vascular elastic arterial extracellular matrix and is associated with age and sex. Therefore, the age and sex of the individual should be taken into account when PP and PWV are used to evaluate arterial stiffness. In the present study, we aimed to determine the combined effect of PP and baPWV on the incidence of new-onset diabetes using data from the Kailuan Study.

\section{RESEARCH DESIGN AND METHODS \\ Study participants}

The Kailuan Study is a longitudinal cohort study of current and retired employees of the Kailuan Group in Tangshan city, China. Between 2006 and 2007, the first health evaluations of employees and retirees of the Kailuan Group were conducted at Kailuan General Hospital and its 11 associated hospitals, and they were followed up every 2 years. A total of 101510 participants were enrolled for the baseline survey. All the participants completed a standardized questionnaire and underwent physical examinations and laboratory testing. ${ }^{910}$ Between 2010 and 2011, baPWV was measured in some participants, and the full cohort underwent routine follow-up examinations. A total of 21529 individuals participated in the follow-up evaluations between 2010 and 2015 and underwent baPWV measurement for the present study. Participants with diabetes $(\mathrm{n}=2795)$ or atrial fibrillation $(n=47)$ were excluded, as well as those for whom blood pressure data were missing $(\mathrm{n}=12)$, and those who were lost to follow-up ( $\mathrm{n}=56$ ), which left data from 18619 participants for analysis (figure 1). The study was performed according to the principles of the Declaration of Helsinki. Written informed consent was obtained from all the participants.

\section{Measurement of blood pressure}

Blood pressure was measured in the right brachial artery using a calibrated mercury column sphygmomanometer three times at intervals of 1-2 min, after the participants had rested for at least $5 \mathrm{~min}$. If the difference between two values was $>5 \mathrm{~mm} \mathrm{Hg}$, the blood pressure was remeasured. The mean of the three values was recorded. The HEM8102A electronic sphygmomanometers used, which were

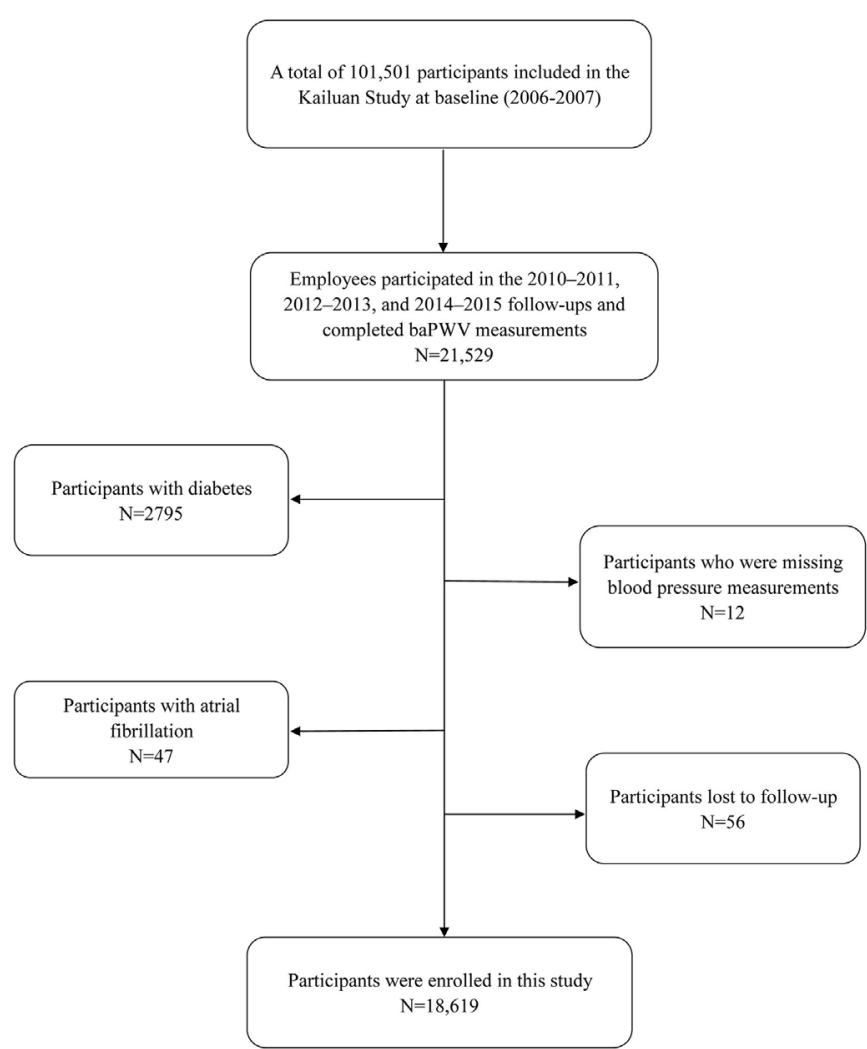

Figure 1 Flow chart for the inclusion of participants in the study. baPWV, brachial-ankle pulse wave velocity.

manufactured by Omron Co, Ltd (Dalian, China), have been used since 2014. PP was calculated as the difference between the systolic and diastolic pressure. Mean aterial pressure (MAP) was calculated as the diastolic pressure plus one third pulse pressure.

\section{Measurement of baPWV}

BaPWV was measured between 07:00 and 09:00 hours on the day of the follow-up examination by trained professionals. The examination room temperature was kept between $22^{\circ} \mathrm{C}$ and $25^{\circ} \mathrm{C}$. A BP-203RPEIII network arterial stiffness detection device (Omron Health Medical Co, Ltd) was used to make measurements in all four limbs, from which baPWV was calculated. In this study, baPWV was calculated as $\mathrm{La}-\mathrm{Lb} / \Delta \mathrm{T}$, in which $\mathrm{La}$ is the distance from the heart to the ankle, $\mathrm{Lb}$ is the distance from the heart to the brachium, and $\Delta \mathrm{T}$ is the time interval between the wavefront of the brachial waveform and that of the ankle waveform. Participants were required to rest for at least $5 \mathrm{~min}$ beforehand, were not permitted to smoke, and had to lie down during the measurements. Bilateral measurements were made twice in each individual and the higher value (left or right) made on the second occasion was recorded as the baPWV. ${ }^{11}{ }^{12}$ In a validation study, three measurements were made three times in 77 participants, following the standard procedure. We calculated the intraclass correlation coefficient (ICC) to assess test-retest reliability; all the pairs showed good (ICC $\geq 0.40)$ to excellent (ICC $\geq 0.75$ ) reliability. 


\section{Assessment of diabetes status}

All the participants provided venous blood samples after an overnight fast of 8-12 hours. Fasting blood glucose (FBG) concentration was measured using the hexokinase/glucose-6-phosphate dehydrogenase method on an autoanalyzer (Hitachi 747; Hitachi, Tokyo, Japan) in the central laboratory of Kailuan General Hospital. The coefficient of variation, calculated using blind quality control specimens, was $<2.0 \%$. Information regarding treatment with insulin or oral hypoglycemic agents was collected during follow-up.

Incident diabetes was defined as a self-reported physician diagnosis of diabetes during the follow-up period, the use of glucose-lowering drugs during the follow-up period, or a change in FBG from $<7.0 \mathrm{mmol} / \mathrm{L}$ at baseline to $\geq 7.0 \mathrm{mmol} / \mathrm{L}$ at the final visit. Patients with pre-existing diabetes, defined as a self-reported a history of diabetes mellitus, the administration of antidiabetic medication, or an FBG $\geq 7.0 \mathrm{mmol} / \mathrm{L}$ at baseline, were excluded. The participants were followed from the day their baPWV was measured until a diagnosis of incident diabetes was made, they were lost to follow-up because of emigration or death, or the end of the follow-up period.

\section{Assessment of covariates}

Epidemiologic, anthropometric, biochemical, and lifestyle survey data for the participants have been published previously. ${ }^{9} 10$ Information regarding their age, sex, smoking habits, alcohol intake, salt intake, physical activity, income, educational level, and medical history, including the presence of hypertension or diabetes, and the use of medication, such as hypoglycemic agents, antihypertensive agents, lipid-lowering agents, or aspirin, was collected using questionnaires. The height, body mass, and heart rate of the participants were measured by a nurse. Body mass index (BMI) was calculated as body mass in kilograms divided by the square of height in meters. Serum total cholesterol, triglyceride, highdensity lipoprotein-cholesterol, low-density lipoproteincholesterol, and high-sensitivity $\mathrm{C}$ reactive protein (hs-CRP) concentrations were measured using an automatic analyzer (Hitachi 747; Hitachi, Tokyo, Japan) in the Central Laboratory of Kailuan Hospital.

\section{Statistical analysis}

Baseline data were collected during the visit at which baPWV was measured. As in a previous study, ${ }^{6}$ the participants were allocated to four groups according to their PP and baPWV, which were categorised as normal or high on the basis of age-specific and sex-specific median values: (1) a normal PP/baPWV group; (2) a normal PP/high baPWV group; (3) a high PP/normal baPWV group; and (4) a high PP/baPWV group. The relationship between baPWV and PP was evaluated using Pearson's correlation coefficient. The cumulative incidence of diabetes in each group was calculated using the Kaplan-Meier method and was also compared using the log-rank test.
Multivariable Cox proportional hazards models were used to evaluate the relationships between the four groups and the incidence of diabetes and to evaluate the individual relationships between PP or baPWV and the incidence of diabetes. We determined whether there was a statistical interaction between the exposure categories (normal or high PP and normal or high baPWV) by entering these variables as interaction terms into the multivariable models. We adjusted for age, sex, BMI, mean arterial pressure, serum hs-CRP concentration, smoking status, alcohol consumption status, physical activity, hyperlipidemia, family history of diabetes, and the use of antihypertensive agents in the multivariable models. The covariates were selected on the basis of previous reports of their association with diabetes in China. ${ }^{43-15}$

Several sensitivity analyses were performed to test the robustness of the results. Because some antihypertensive agents, for example, diuretics and some $\beta$-blockers, and lipid-lowering agents can adversely affect glucose metabolism, we excluded participants who used cholesterollowering agents and antihypertensive agents during the follow-up period. We also excluded individuals with ankle-brachial index $\leq 0.9$ at baseline, because arterial stenosis or occlusion in a lower extremity can affect the accuracy of baPWV measurements. We also performed subgroup analyses according to sex and age. To assess the potential confounding effects of pre-diabetes and gestational diabetes, we performed two sensitivity analyses that separately excluded pre-diabetic patients and gestational diabetes. SAS V.9.4 (Cary, North Carolina, USA) was used for statistical analyses, and a two-sided $p$ value of $<0.05$ was considered to represent statistical significance.

\section{RESULTS}

Table 1 shows the baseline characteristics of each group. The mean age of the participants was 47.65 (SD: 12.32) years, and $12458(66.91 \%)$ of them were male. The median PP and baPWV were $44.67 \mathrm{~mm} \mathrm{Hg}$ and 1405.00 $\mathrm{cm} / \mathrm{s}$, respectively. The age-specific and sex-specific median PP and baPWV values are shown in online supplemental table 1 . There were 7488 participants (40.22\%) who were discordant with respect to PP and baPWV status (3506 with normal PP and high baPWV, and 3982 with high PP and normal baPWV). The correlation coefficient for the relationship between PP and baPWV was 0.33 after adjustment for age and sex.

\section{Cumulative incidence of diabetes}

The length of the follow-up period was $4.27 \pm 1.91$ years, and the number of follow-up visits was $2.94 \pm 0.89$. During this period, a total of 877 participants developed diabetes, yielding a cumulative incidence of $8.86 \%$ and an incidence density of $11.03 / 1000$ persons per year. The cumulative incidences for the normal and high PP groups were $8.82 \%$ and $10.75 \%$, and those for the normal baPWV and high baPWV groups were $6.61 \%$ and $12.86 \%$, respectively. The cumulative incidences of diabetes were $6.53 \%$, 
Table 1 Baseline characteristics of participants by PP and baPWV status

\begin{tabular}{|c|c|c|c|c|c|c|}
\hline & $\begin{array}{l}\text { Total population } \\
\mathrm{n}=18619\end{array}$ & $\begin{array}{l}\text { Normal PP and } \\
\text { baPWV } \\
n=5295\end{array}$ & $\begin{array}{l}\text { Normal PP and } \\
\text { increased baPWV } \\
n=3506\end{array}$ & $\begin{array}{l}\text { Increased PP and } \\
\text { normal baPWV } \\
\mathrm{n}=3982\end{array}$ & $\begin{array}{l}\text { Increased PP } \\
\text { and baPWV } \\
\mathrm{n}=5836\end{array}$ & $P$ value \\
\hline Age (year) & $47.65 \pm 12.32$ & $47.99 \pm 12.26$ & $46.89 \pm 12.62$ & $47.01 \pm 12.34$ & $48.24 \pm 12.13$ & $<0.001$ \\
\hline Male (\%) & $12458(66.91)$ & $3644(68.82)$ & $2437(69.51)$ & $2563(64.36)$ & 3814 (65.35) & $<0.001$ \\
\hline $\mathrm{SBP}(\mathrm{mm} \mathrm{Hg})$ & $127.96 \pm 18.07$ & $115.62 \pm 12.55$ & $121.76 \pm 13.34$ & $131.07 \pm 15.07$ & $140.75 \pm 17.49$ & $<0.001$ \\
\hline $\mathrm{DBP}(\mathrm{mm} \mathrm{Hg})$ & $81.70 \pm 10.91$ & $78.59 \pm 9.56$ & $83.67 \pm 10.59$ & $78.77 \pm 10.35$ & $85.32 \pm 11.25$ & $<0.001$ \\
\hline $\mathrm{PP}(\mathrm{mm} \mathrm{Hg})$ & $46.26 \pm 12.52$ & $37.03 \pm 6.97$ & $38.09 \pm 6.71$ & $52.30 \pm 9.73$ & $55.42 \pm 11.77$ & $<0.001$ \\
\hline $\mathrm{BaPWV}(\mathrm{cm} / \mathrm{s})$ & $1466.99 \pm 321.18$ & $1283.23 \pm 181.77$ & $1602.27 \pm 303.47$ & $1293.32 \pm 184.92$ & $1670.95 \pm 341.60$ & $<0.001$ \\
\hline $\mathrm{BaPWV} \geq 1.4 \mathrm{k} \mathrm{cm} / \mathrm{s}(\%)$ & $9489(50.96)$ & 1079 (20.38) & $2747(78.35)$ & $870(21.85)$ & $4793(82.13)$ & $<0.001$ \\
\hline $\mathrm{BMI}\left(\mathrm{kg} / \mathrm{m}^{2}\right)$ & $24.71 \pm 3.36$ & $24.27 \pm 3.28$ & $24.61 \pm 3.35$ & $24.75 \pm 3.24$ & $25.13 \pm 3.44$ & $<0.001$ \\
\hline $\mathrm{FBG}(\mathrm{mmol} / \mathrm{L})$ & $5.19 \pm 0.67$ & $5.10 \pm 0.58$ & $5.19 \pm 0.88$ & $5.19 \pm 0.60$ & $5.28 \pm 0.63$ & $<0.001$ \\
\hline $\mathrm{TC}(\mathrm{mmol} / \mathrm{L})$ & $5.02 \pm 1.60$ & $4.94 \pm 1.87$ & $5.00 \pm 1.32$ & $4.99 \pm 1.38$ & $5.13 \pm 1.62$ & $<0.001$ \\
\hline $\mathrm{TG}(\mathrm{mmol} / \mathrm{L})$ & $1.22(0.84-1.84)$ & $1.14(0.80-1.68)$ & $1.28(0.89-2.00)$ & $1.13(0.78-1.71)$ & $1.32(0.90-2.01)$ & $<0.001$ \\
\hline LDL-C (mmol/L) & $2.64 \pm 0.90$ & $2.56 \pm 0.92$ & $2.63 \pm 1.04$ & $2.64 \pm 0.81$ & $2.72 \pm 0.83$ & $<0.001$ \\
\hline $\mathrm{HDL}-\mathrm{C}(\mathrm{mmol} / \mathrm{L})$ & $1.50 \pm 0.71$ & $1.50 \pm 0.45$ & $1.49 \pm 0.71$ & $1.50 \pm 0.66$ & $1.50 \pm 0.90$ & 0.822 \\
\hline hs-CRP (mg/L) ${ }^{*}$ & $-0.55 \pm 2.62$ & $-0.57 \pm 2.45$ & $-0.47 \pm 2.56$ & $-0.65 \pm 2.71$ & $-0.51 \pm 2.74$ & 0.012 \\
\hline Smoking (\%) & $6708(36.03)$ & 1943 (36.69) & $1362(38.85)$ & 1300 (32.65) & $2103(36.03)$ & 0.002 \\
\hline Drinking (\%) & $5727(30.76)$ & 1709 (32.28) & $1186(33.83)$ & $1080(27.12)$ & $1752(30.02)$ & 0.007 \\
\hline Exercise (\%) & $1687(9.06)$ & $565(10.67)$ & $316(9.01)$ & $331(8.31)$ & $475(8.14)$ & $<0.001$ \\
\hline Hypertension (\%) & $6551(35.18)$ & $968(18.28)$ & $1211(34.54)$ & $1220(30.64)$ & $3152(54.01)$ & \\
\hline $\begin{array}{l}\text { Blood pressure lowering } \\
\text { agents (\%) }\end{array}$ & $2311(12.41)$ & $321(6.06)$ & $461(13.15)$ & $361(9.07)$ & $1168(20.01)$ & $<0.001$ \\
\hline Lipid-lowering drugs (\%) & $164(0.88)$ & $36(0.68)$ & $37(1.06)$ & $25(0.63)$ & $66(1.13)$ & 0.013 \\
\hline
\end{tabular}

P, comparison of baseline characteristics between different PP and baPWV groups.

*Log10 transformation.

baPWV, brachial-ankle pulse wave velocity; BMI, body mass index; DBP, diastolic blood pressure; FBG, fasting blood glucose; HDL-C, high-density lipoprotein cholesterol; hs-CRP, high-sensitivity $\mathrm{C}$ reactive protein; LDL-C, low-density lipoprotein cholesterol; PP, pulse pressure; SBP, systolic blood pressure; TC, total cholesterol; TG, triglyceride.

$12.04 \%, 6.24 \%$, and $13.18 \%$ for the normal PP/baPWV, normal PP/high baPWV, high PP/normal baPWV, and high PP/baPWV groups, respectively (figure 2).

\section{Comparison of the risk of new-onset diabetes in each group}

Compared with the normal PP group, the high PP group had a significantly higher risk of developing diabetes, according to model 1 . However, in models 2 and 3, high $\mathrm{PP}$ was not significantly associated with the risk of diabetes (HR 1.08; 95\% CI 0.93 to 1.25) (table 2). Compared with the normal baPWV group, the high baPWV group had a significantly higher risk of diabetes, according to models 1 and 2, and after adjustment for PP in model 3, an association between baPWV and the risk of diabetes remained (HR 1.64; 95\% CI 1.41 to 1.90 ) (table 3 ).

Table 4 shows the risk of diabetes in each of the groups. In the multivariable-adjusted model, the HRs for newonset diabetes were significantly higher in the normal PP/ high baPWV (HR 1.66, 95\% CI 1.35 to 2.05 ) and high PP/ baPWV (HR 1.74, 95\% CI 1.43 to 2.13) groups than in the normal PP/baPWV group. In subgroup analyses, no significant associations were found among female participants alone (online supplemental tables 3-6). In addition, the effect estimates were not significantly affected by the exclusion of patients with pre-diabetes or gestational diabetes (online supplemental tables 7 and 8).

\section{DISCUSSION}

In the present study, we used age-specific and sex-specific cut-off values to define high and normal PP and baPWV and found that high baPWV was associated with a higher risk of diabetes, whereas high PP was not. However, when combinations of high or normal PP and baPWV were considered, we found that participants with both high baPWV and high PP were at the highest risk.

The present results are consistent with those of previous studies that showed that arterial stiffness increases the risk of diabetes. Muhammad $e t a l^{15}$ used carotid-femoral pulse wave velocity (cfPWV) as an indicator of arterial stiffness and found that the risk of diabetes in people with high cfPWV was 3.24 times higher than in those with low cfPWV. In addition, a prospective study of 2429 hypertensive patients showed that baseline baPWV is positively associated with the risk of developing diabetes. ${ }^{5}$ More recently, Liu et $a l^{16}$ found that there is a dose-response 


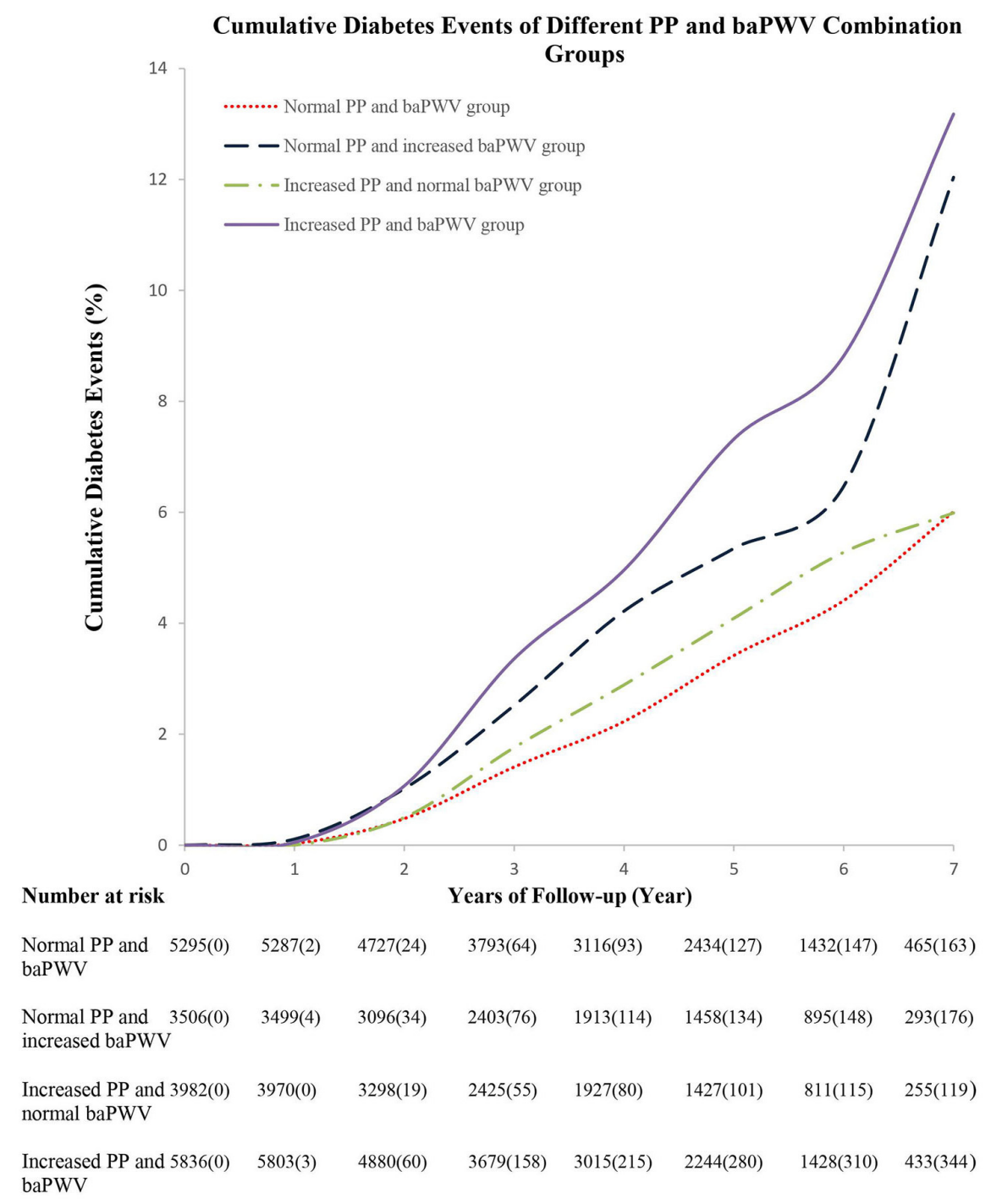

Figure 2 Cumulative diabetes incidence in groups with combinations of normal or high PP and baPWV. $\mathrm{p}<0.05$; log-rank test. baPWV, brachial-ankle pulse wave velocity; PP, pulse pressure.

relationship between baPWV and the risk of type 2 diabetes, and in a prospective cohort study conducted in China, it was shown that baPWV might be an independent predictor of new-onset type 2 diabetes. ${ }^{17}$ Consistent with these previous findings, the present results show that there is a 1.64-fold increase in the risk of developing diabetes in patients with a high baPWV.
Although several plausible biological pathways have been implicated, the mechanism underlying the link between baPWV and diabetes remains unclear. First, endothelial cell dysfunction could cause microvascular dysfunction, ${ }^{18}$ leading to poor tissue perfusion, impaired glucose metabolism and insulin resistance, and arterial stiffness. ${ }^{19-21}$ In addition, arterial stiffness might worsen

\begin{tabular}{|c|c|c|c|c|c|c|}
\hline \multirow[b]{2}{*}{ Groups } & \multirow[b]{2}{*}{$\mathbf{N}$} & \multirow[b]{2}{*}{ Events } & \multirow{2}{*}{$\begin{array}{l}\text { Incidence density } \\
\text { (persons per year) }\end{array}$} & \multirow{2}{*}{$\begin{array}{l}\text { Model } 1 \\
\text { HR }(95 \% \mathrm{Cl})\end{array}$} & \multirow{2}{*}{$\begin{array}{l}\text { Model } 2 \\
\text { HR }(95 \% \mathrm{Cl})\end{array}$} & \multirow{2}{*}{$\begin{array}{l}\text { Model } 3 \\
\text { HR }(95 \% \mathrm{Cl})\end{array}$} \\
\hline & & & & & & \\
\hline Normal PP & 8801 & 379 & 9.70 & Ref. & Ref. & Ref. \\
\hline Increased PP & 9818 & 498 & 12.31 & $1.41(1.23$ to 1.61$)$ & $1.13(0.97$ to 1.31$)$ & 1.08 (0.93 to 1.25$)$ \\
\hline Per SD increase in PP & 18619 & 877 & 11.03 & 1.20 (1.13 to 1.28$)$ & 1.06 (0.98 to 1.15$)$ & 1.01 (0.93 to 1.09$)$ \\
\hline
\end{tabular}

Model 1: adjusted for age and gender; model 2, adjusted for age, gender, BMI, MAP, hs-CRP, smoking, drinking, exercise, hyperlipidemia, family history of diabetes and blood pressure lowering agents; model 3: adjusted for all the variables in model 2 and baPWV.

baPWV, brachial-ankle pulse wave velocity; BMl, body mass index; hs-CRP, high-sensitivity C reactive protein; MAP, mean arterial pressure; PP, pulse pressure. 
Table 3 Risk of increased baPWV on the new-onset diabetes events

\begin{tabular}{|c|c|c|c|c|c|c|}
\hline \multirow[b]{2}{*}{ Groups } & \multirow[b]{2}{*}{$\mathbf{N}$} & \multirow[b]{2}{*}{ Events } & \multirow{2}{*}{$\begin{array}{l}\text { Incidence density } \\
\text { (persons per year) }\end{array}$} & \multirow{2}{*}{$\begin{array}{l}\text { Model } 1 \\
\text { HR }(95 \% \mathrm{Cl})\end{array}$} & \multirow{2}{*}{$\begin{array}{l}\text { Model } 2 \\
\text { HR }(95 \% \mathrm{Cl})\end{array}$} & \multirow{2}{*}{$\begin{array}{l}\text { Model } 3 \\
\text { HR }(95 \% \mathrm{Cl})\end{array}$} \\
\hline & & & & & & \\
\hline Normal baPWV & 9277 & 306 & 7.65 & Ref. & Ref. & Ref. \\
\hline Increased baPWV & 9342 & 571 & 14.44 & 1.96 (1.70 to 2.25$)$ & $1.65(1.42$ to 1.91$)$ & $1.64(1.41$ to 1.90$)$ \\
\hline Per SD increase in baPWV & 18619 & 877 & 11.03 & 1.41 (1.33 to 1.49$)$ & $1.36(1.27$ to 1.46$)$ & $1.36(1.27$ to 1.46$)$ \\
\hline
\end{tabular}

Model 1: adjusted for age and gender; model 2: adjusted for age, gender, BMI, MAP, hs-CRP, smoking, drinking, exercise, hyperlipidemia, family history of diabetes, blood pressure lowering agents; model 3, adjusted for all the variables in model 2 and PP.

baPWV, brachial-ankle pulse wave velocity; BMI, body mass index; hs-CRP, high-sensitivity C reactive protein; MAP, mean arterial pressure; PP, pulse pressure.

microvascular lesions, leading to the creation of a vicious circle. $^{22}{ }^{23}$ Second, whereas normal arteries buffer the PP, arterial stiffness leads to high blood flow through low-resistance organs, such as the brain and kidney, resulting in organ dysfunction. ${ }^{24}$ Furthermore, because the pancreas is a low-resistance, high-blood flow organ (mean tissue perfusion $250-300 \mathrm{~mL} / \mathrm{min} / 100 \mathrm{~g}$ ), it may be that its endocrine function is adversely affected by arterial stiffness.

We did not find that high PP is associated with a higher risk of diabetes, which is consistent with the finding of Chen $e t a l^{25}$ that PP was not associated with the risk of diabetes in a hypertensive population. In contrast, Yasuno $e t a t^{4}$ found that PP is an independent predictor of new-onset diabetes in hypertensive patients in Japan. However, it is important to highlight that the present study was performed in the general population and that we recruited participants of a relatively young age (mean age 47 years). Chen $e t$ al also studied young hypertensive patients (mean age 41 years), whereas the mean age of the participants in the study by Yasuno et al was 64 years. In addition, we included the direct measures of arterial stiffness as a covariate in the analysis. These factors might explain the difference in the findings of the present study and that of Yasuno et al.

The correlation coefficient for the relationship between PP and baPWV was 0.33 after adjustment for age and sex. Kang $e t a l^{26}$ and Kim $e t a l^{27}$ made similar findings and obtained correlation coefficients of 0.44 and 0.36 , respectively. In another study that used cfPWV as the measure of arterial stiffness, the correlation coefficient was $0.43,{ }^{6}$ which implies that there is a difference between the use of PP and cfPWV for the evaluation of arterial stiffness. Furthermore, this study showed that the effects of cfPWV and PP on the risk of cardiovascular disease are additive, ${ }^{6}$ which is similar to the findings of the present study, in which the presence of both high PP and high baPWV further increased the risk of incident diabetes. We also found that individuals with normal PP and high baPWV and those with high PP and baPWV had higher risks of diabetes than those with normal PP and baPWV. However, individuals with high PP and normal baPWV did not have a significantly higher risk of diabetes. These results suggest that baPWV may be a better predictor of diabetes than PP.

The present study had several limitations. First, we used baPWV, rather than cfPWV, as a measure of arterial stiffness. However, baPWV closely correlates with cfPWV and has been widely used in studies of Asian populations. ${ }^{28}{ }^{29}$ Second, a single population was studied; therefore, the results require validation in other populations. Third, diagnoses of diabetes were made on the basis of single measurements of FBG, which may have resulted in an underestimation of the incidence of diabetes. Finally, we did not differentiate patients with type 1 and type 2 diabetes. However, according to the Chinese clinical guidelines for the prevention of diabetes, type 2 diabetes represents about $95 \%$ of all cases. ${ }^{30}$

In summary, we have shown that high baPWV is associated with a higher risk of diabetes, and individuals with both a high baPWV and a high PP have a still higher risk of diabetes. The measurement of baPWV in the general population may significantly contribute to the prevention of diabetes.

Table 4 Risk of different PP and baPWV combination on the new-onset diabetes events

\begin{tabular}{|c|c|c|c|c|c|}
\hline \multirow[b]{2}{*}{ Groups } & \multirow[b]{2}{*}{$\mathbf{N}$} & \multirow[b]{2}{*}{ Events } & \multirow{2}{*}{$\begin{array}{l}\text { Incidence density } \\
\text { (persons per year) }\end{array}$} & \multirow{2}{*}{$\begin{array}{l}\text { Model } 1 \\
\text { HR }(95 \% \mathrm{Cl})\end{array}$} & \multirow{2}{*}{$\begin{array}{l}\text { Model } 2 \\
\text { HR }(95 \% \mathrm{Cl})\end{array}$} \\
\hline & & & & & \\
\hline Normal PP and baPWV & 5295 & 178 & 7.46 & Ref. & Ref. \\
\hline Normal PP and increased baPWV & 3506 & 201 & 13.21 & 1.88 (1.53 to 2.30$)$ & 1.66 (1.35 to 2.05$)$ \\
\hline Increased PP and normal baPWV & 3982 & 128 & 7.94 & 1.23 (0.98 to 1.54$)$ & $1.09(0.86$ to 1.37$)$ \\
\hline Increased PP and baPWV & 5836 & 370 & 15.21 & 2.29 (1.91 to 2.73$)$ & $1.74(1.43$ to 2.13$)$ \\
\hline
\end{tabular}

Model 1: adjusted for age and gender; model 2: adjusted for age, gender, BMI, MAP, hs-CRP, smoking, drinking, exercise, hyperlipidemia, family history of diabetes and blood pressure lowering agents.

baPWV, brachial-ankle pulse wave velocity; BMI, body mass index; hs-CRP, high-sensitivity C reactive protein; MAP, mean arterial pressure; PP, pulse pressure. 
Author affiliations

'Department of Cardiology, Kailuan General Hospital, North China University of Science and Technology, Tangshan, China

${ }^{2}$ Graduate School, North China University of Science and Technology, Tangshan, China

${ }^{3}$ Department of Maternal and Child Health, School of Public Health, Tongji Medical College, Huazhong University of Science and Technology, Wuhan, China

Acknowledgements We would like to thank all of the staff members in the medical health check-up center at Kailuan Hospital, and we thank Mark Cleasby, $\mathrm{PhD}$, from Liwen Bianji, Edanz Group China (www.liwenbianji.cn/ac), for editing the English text of a draft of this manuscript.

Contributors $\mathrm{XH}$ and SW designed the study. YWu and XH performed statistical analysis and drafted the manuscript with assistance from YT. JG, YWa, CZ, ZH, AX, $\mathrm{SC}, \mathrm{YM}, \mathrm{MZ}$ and QL contributed to the specification of the analyses and critically reviewed and edited the manuscript. YWu and XH are the guarantors of this work and, as such, had full access to all of the data in the study and takes responsibility for the integrity of the data and the accuracy of the data analysis.

Funding The authors have not declared a specific grant for this research from any funding agency in the public, commercial or not-for-profit sectors.

Competing interests None declared.

Patient consent for publication Not required.

Ethics approval This study was approved by the Ethics Committee of the Kailuan General Hospital (ChiCTR-TNC-11001489).

Provenance and peer review Not commissioned; externally peer reviewed.

Data availability statement № data are available. Proposals for collaborative research can be submitted to Wu(drwus|@163.com)

Supplemental material This content has been supplied by the author(s). It has not been vetted by BMJ Publishing Group Limited (BMJ) and may not have been peer-reviewed. Any opinions or recommendations discussed are solely those of the author(s) and are not endorsed by BMJ. BMJ disclaims all liability and responsibility arising from any reliance placed on the content. Where the content includes any translated material, BMJ does not warrant the accuracy and reliability of the translations (including but not limited to local regulations, clinical guidelines, terminology, drug names and drug dosages), and is not responsible for any error and/or omissions arising from translation and adaptation or otherwise.

Open access This is an open access article distributed in accordance with the Creative Commons Attribution Non Commercial (CC BY-NC 4.0) license, which permits others to distribute, remix, adapt, build upon this work non-commercially, and license their derivative works on different terms, provided the original work is properly cited, appropriate credit is given, any changes made indicated, and the use is non-commercial. See: http://creativecommons.org/licenses/by-nc/4.0/.

ORCID iDs

Yaohua Tian http://orcid.org/0000-0002-9853-6991

Shouling Wu http://orcid.org/0000-0001-7095-6022

\section{REFERENCES}

1 International Diabetes Federation. IDF diabetes atlas. 9th edn. Brussels: International Diabetes Federation, 2019. http://www. diabetesatlas.org

2 Wang L, Gao P, Zhang M, et al. Prevalence and ethnic pattern of diabetes and prediabetes in China in 2013. JAMA 2017:317:2515-23.

3 Weisman A, Fazli GS, Johns A, et al. Evolving trends in the epidemiology, risk factors, and prevention of type 2 diabetes: a review. Can J Cardiol 2018;34:552-64.

4 Yasuno S, Ueshima K, Oba K, et al. Is pulse pressure a predictor of new-onset diabetes in high-risk hypertensive patients?: a subanalysis of the candesartan antihypertensive survival evaluation in Japan (CASE-J) trial. Diabetes Care 2010;33:1122-7.

5 Zhang Y, He P, Li Y, et al. Positive association between baseline brachial-ankle pulse wave velocity and the risk of new-onset diabetes in hypertensive patients. Cardiovasc Diabetol 2019;18:111.

6 Niiranen TJ, Kalesan B, Mitchell GF, et al. Relative contributions of pulse pressure and arterial stiffness to cardiovascular disease. Hypertension 2019;73:712-7.
7 Nürnberger J, Dammer S, Opazo Saez A, et al. Diastolic blood pressure is an important determinant of augmentation index and pulse wave velocity in young, healthy males. J Hum Hypertens 2003;17:153-8.

8 Lu Y-C, Lyu P, Zhu H-Y, et al. Brachial-ankle pulse wave velocity compared with mean arterial pressure and pulse pressure in risk stratification in a Chinese population. $J$ Hypertens 2018;36:528-36.

9 Wu Z, Jin C, Vaidya A, et al. Longitudinal patterns of blood pressure, incident cardiovascular events, and all-cause mortality in normotensive diabetic people. Hypertension 2016;68:71-7.

10 Zhang Q, Zhou Y, Gao X, et al. Ideal cardiovascular health metrics and the risks of ischemic and intracerebral hemorrhagic stroke. Stroke 2013;44:2451-6.

11 Song $Y, X u B, X u R$, et al. Independent and joint effect of brachialankle pulse wave velocity and blood pressure control on incident stroke in hypertensive adults. Hypertension 2016;68:46-53.

12 Lee SJ, Avolio A, Seo DC, et al. Relationship between brachialankle pulse wave velocity and incident hypertension according to 2017 ACC/AHA high blood pressure guidelines. J Am Heart Assoc 2019;8:e013019.

13 Donath MY, Shoelson SE. Type 2 diabetes as an inflammatory disease. Nat Rev Immunol 2011;11:98-107.

$14 \mathrm{Hu}$ C, Jia W. Diabetes in China: epidemiology and genetic risk factors and their clinical utility in personalized medication. Diabetes 2018;67:3-11.

15 Muhammad IF, Borné Y, Östling G, et al. Arterial stiffness and incidence of diabetes: a population-based cohort study. Diabetes Care 2017:40:1739-45.

16 Liu Y, Lai X, Guo W, et al. Total white blood cell count mediated the association between increased arterial stiffness and risk of type 2 diabetes mellitus in Chinese adults. Arterioscler Thromb Vasc Biol 2020;40:1009-15.

17 Lou Y-M, Liao M-Q, Wang C-Y, et al. Association between brachial-ankle pulse wave velocity and risk of type 2 diabetes mellitus: results from a cohort study. BMJ Open Diabetes Res Care 2020;8:e001317.

18 Muris DMJ, Houben AJHM, Schram MT, et al. Microvascular dysfunction is associated with a higher incidence of type 2 diabetes mellitus: a systematic review and meta-analysis. Arterioscler Thromb Vasc Biol 2012;32:3082-94

19 Levy BI, Schiffrin EL, Mourad J-J, et al. Impaired tissue perfusion: a pathology common to hypertension, obesity, and diabetes mellitus. Circulation 2008;118:968-76.

20 Vincent MA, Clerk LH, Lindner JR, et al. Microvascular recruitment is an early insulin effect that regulates skeletal muscle glucose uptake in vivo. Diabetes 2004;53:1418-23.

21 Cooper LL, Palmisano JN, Benjamin EJ, et al. Microvascular function contributes to the relation between aortic stiffness and cardiovascular events: the Framingham heart study. Circ Cardiovasc Imaging 2016;9:e004979.

22 Malik AR, Kondragunta V, Kullo IJ. Forearm vascular reactivity and arterial stiffness in asymptomatic adults from the community. Hypertension 2008;51:1512-8.

23 O'Rourke MF, Hashimoto J. Mechanical factors in arterial aging: a clinical perspective. J Am Coll Cardiol 2007:50:1-13.

24 Ungvari Z, Csiszar A, Kaminski PM, et al. Chronic high pressureinduced arterial oxidative stress: involvement of protein kinase C-dependent $\mathrm{NAD}(\mathrm{P}) \mathrm{H}$ oxidase and local renin-angiotensin system. Am J Pathol 2004;165:219-26.

25 Chen J-Y, Chou C-H, Lee YL, et al. Association of central aortic pressures indexes with development of diabetes mellitus in essential hypertension. Am J Hypertens 2010;23:1069-73.

26 Kang J, Kim H-L, Lim W-H, et al. Relationship between brachialankle pulse wave velocity and invasively measured aortic pulse pressure. J Clin Hypertens 2018;20:462-8.

27 Kim EJ, Park CG, Park JS, et al. Relationship between blood pressure parameters and pulse wave velocity in normotensive and hypertensive subjects: invasive study. J Hum Hypertens 2007:21:141-8.

28 Wu S, Jin C, Li S, et al. Aging, arterial stiffness, and blood pressure association in Chinese adults. Hypertension 2019;73:893-9.

29 Ohkuma T, Ninomiya T, Tomiyama $\mathrm{H}$, et al. Brachial-ankle pulse wave velocity and the risk prediction of cardiovascular disease: an individual participant data meta-analysis. Hypertension 2017;69:1045-52.

30 Chinese Diabetes Society. Clinical guideline for prevention and treatment of type 2 diabetes in China. Chinese $J$ Diabet 2014;22:2-42. 